\title{
Object detection utilizing modified auto encoder and convolutional neural networks
}

\begin{abstract}
Deep learning models are widely used in object detection area, including combination of multiple non-linear data transformations. The objective is receiving brief and concise information for feature representations. Due to the high volume of processing data, object detection in videos has been faced with big challenges, such as mass calculation. To increase the object detection precision in videos, a hybrid method is proposed, in this paper. Some modifications are applied to auto encoder neural networks, for the compact and discriminative learning of object features. Furthermore, for object classification, firstly extracted features are transferred to a convolutional neural network, and after feature convolution with input pictures, they will be classified. The proposed method has two main advantages over other unsupervised feature learning techniques. Firstly, as it will be shown, features are detected with a much higher precision. Secondly, in the proposed method, the outcome is compact and additional unnecessary information is removed; while the existing unsupervised feature learning models mainly learn repeated and redundant information of the features. Experimental evaluation shows that precision of feature detection improved by $1.5 \%$ in average in compare with the state-of-the-art methods.
\end{abstract}

Keyword: Deep learning; Object detection; Classification; Unsupervised feature learning 